\title{
LIF Measurements on an Atomic Helium Beam in the Edge of a Fusion Plasma - possible derivation of the electron density
}

\author{
Maciej Krychowiak ${ }^{\mathrm{a}}, \mathrm{Ph}$. Mertens $^{\mathrm{b}}$, B. Schweer ${ }^{\mathrm{b}}$, S. Brezinsek $^{\mathrm{b}}$, R. König ${ }^{\mathrm{a}}$, \\ O. Schmitz ${ }^{\mathrm{b}}$, M. Brix ${ }^{\mathrm{c}}$, T. Klinger ${ }^{\mathrm{a}}$ and U. Samm ${ }^{\mathrm{b}}$ \\ ${ }^{a}$ Max-Planck-Institut für Plasmaphysik, EURATOM-Association, Wendelsteinstraße 1, D-17491 Greifswald, \\ Germany, \\ ${ }^{b}$ Institut für Energieforschung (IEF 4-Plasmaphysik), FZ Jülich, EURATOM-Association, TEC, Wilhelm-Johnen-Straße \\ D-52428 Jülich, Germany \\ ${ }^{c}$ UKEA, JET-Experimental Department, EURATOM-Association, Culham Science Centre, ABINGDON, \\ Oxfordshire, OX14 3DB, U.K.
}

\begin{abstract}
Local values of the electron density and temperature in the edge of a fusion plasma can be derived with high space and time resolution by the use of line radiation of atomic helium beams. The accuracy of this method is mainly limited by the uncertainties in the collisional-radiative model which is needed in order to obtain both plasma parameters from the measured relative intensities of atomic helium lines. Combination of a helium beam with a pulsed high-power laser provides a possibility of $n_{\mathrm{e}}$ measurement which does not require a detailed knowledge of the collisional-radiative model. The method relies on resonant laser pumping of some levels and analyzing their fluorescence after the end of the laser pulse. Such measurements were already performed in low temperature plasmas with some content of atomic helium $[1,2,3]$. In this paper, we discuss the applicability of this method in the fusion edge plasma in the density range of $\sim 10^{12}-10^{13} \mathrm{~cm}^{-3}$ when exciting helium atoms with a laser at the wavelength of $\lambda=388.9 \mathrm{~nm}$ tuned to the triplet transition $2^{3} \mathrm{~S} \rightarrow 3^{3} \mathrm{P}^{\mathrm{o}}$ and observing the fluorescence light at the laser wavelength and at $\lambda=587.6 \mathrm{~nm}\left(3^{3} \mathrm{D} \rightarrow 2^{3} \mathrm{P}^{0}\right)$. A first test measurement at the TEXTOR tokamak in Jülich performed by use of an excimer-pumped dye laser in connection with a thermal helium beam is shown and discussed.
\end{abstract}

Keywords: laser-induced fluorescence, electron density measurement, fusion edge plasma PACS: 52.70.Kz

\section{INTRODUCTION}

The knowledge of the electron density and temperature with good time and space resolution is crucial for the understanding and modelling of the plasma edge of fusion experiments. It is not only important because of the interplay between the edge and the bulk plasma but also due to a strong impact of the edge plasma on the plasmafacing components. Different methods are applied in order to measure both these plasma parameters, such as Langmuir probes, Thomson scattering of laser light or atomic lithium and helium beams [4]. The accuracy of the last mentioned method strongly relies on the knowledge of the collisional-radiative model for atomic helium which must be applied in order to obtain $n_{\mathrm{e}}$ and $T_{\mathrm{e}}$ values from the line intensity ratios [5]. Our initial work was based on laserinduced fluorescence measurements on a thermal helium beam at the TEXTOR tokamak in Jülich [6]. It showed a possibility to derive some rate coefficients for collisional population transfer between excited helium levels. In one triplet measurement scheme the electron density could be derived from the fluorescence signal arising due to collisional coupling between excited levels. 


\section{DERIVATION OF THE ELECTRON DENSITY FROM LASER- AND COLLISION- INDUCED FLUORESCENCE SIGNALS}

Electron density measurements in low temperature plasmas performed by use of the laser-induced fluorescence of helium have been already reported. Chall et al. [1] measured $n_{\mathrm{e}} \approx 5 \times 10^{12} \mathrm{~cm}^{-3}$ from the time decay rate of the fluorescence signal at the laser wavelength at $\lambda=587.6 \mathrm{~nm}$ in an argon arc plasma of $T_{\mathrm{e}} \approx 0.4 \mathrm{eV}$ with small admixture of helium. The electron density in the range $n_{\mathrm{e}}=10^{11}-10^{12} \mathrm{~cm}^{-3}$ in a helium plasma of $T_{\mathrm{e}} \approx 5 \mathrm{eV}$ was determined by Tsuchida et al. [2] from the ratio of time integrated collision-induced $(\lambda=667.8 \mathrm{~nm})$ and laserinduced fluorescence signals upon the laser excitation at $\lambda=501.6 \mathrm{~nm}$ in singlet helium. Shcheglov et al. [3] determined electron densities of $n_{\mathrm{e}} \approx 5 \times 10^{11} \mathrm{~cm}^{-3}$ in an argon plasma $\left(T_{\mathrm{e}} \approx 10 \mathrm{eV}\right)$ with helium content using relative collision-induced signals $\mathrm{I}_{388.9 \mathrm{~nm}} / \mathrm{I}_{706.5 \mathrm{~nm}}$ upon laser excitation at $\lambda=587.6 \mathrm{~nm}$.

For the case of higher temperatures and densities of edge fusion plasmas it is suggested to use the time trace of the collision-induced fluorescence signal at $\lambda=587.6 \mathrm{~nm}$ upon laser excitation at $\lambda=388.9 \mathrm{~nm}$. The high signal-tonoise ratio results from the relatively high population of the triplet metastable in our parameter range and a larger rate coefficient for the collisional transition from the pumped level $3^{3} \mathrm{P}^{\mathrm{o}}$ to the observed level $3^{3} \mathrm{D}$ than e.g. in the opposite direction. Moreover, thanks to the wavelength difference between the laser light and the observed fluorescence, the stray light of the laser does not blind the detectors.

As can be seen in FIGURE 1 a very pure time trace of the collision-induced fluorescence signal at the wavelength $\lambda=587.6 \mathrm{~nm}$, upon laser excitation at the wavelength $\lambda=388.9 \mathrm{~nm}$, can be obtained in situ in the edge plasma of the TEXTOR tokamak. The decay shape of this signal depends mainly on the collisional transition rate between the upper level $3^{3} \mathrm{P}^{\circ}$ and the level $3^{3} \mathrm{D}$ from which the collisional fluorescence is observed. The transition rate is given, provided only electron collisions play a role, by the product of electron density and the rate coefficient. Thus, at known electron density the collisional rate coefficient can be obtained for a given electron temperature. On the other hand, once the rate coefficient has been determined, the decaying fluorescence signal at $\lambda=587.6 \mathrm{~nm}$ can be used to derive the local electron density. In FIGURE 1 (b) we see two additional calculated time traces calculated for a slightly increased and decreased value of the electron density. Both curves are significantly different from the initial calculation. Hence, the derivation of $n_{\mathrm{e}}$ with an accuracy of $\sim 15 \%$ should be possible in this way, provided the value of the mentioned rate coefficient is known with sufficient accuracy. At this point it should be mentioned that the excitation rate coefficient of the considered transition can be affected also by heavy particle collisions (background plasma ions) for which no reliable data exist so far. However, the collision-induced fluorescence at $\lambda=587.6 \mathrm{~nm}$ measures the total rate coefficient, i.e. including the electron and e.g. the proton collision population transfer, for a given plasma density. This total rate coefficient should be measured for different plasma temperature values.
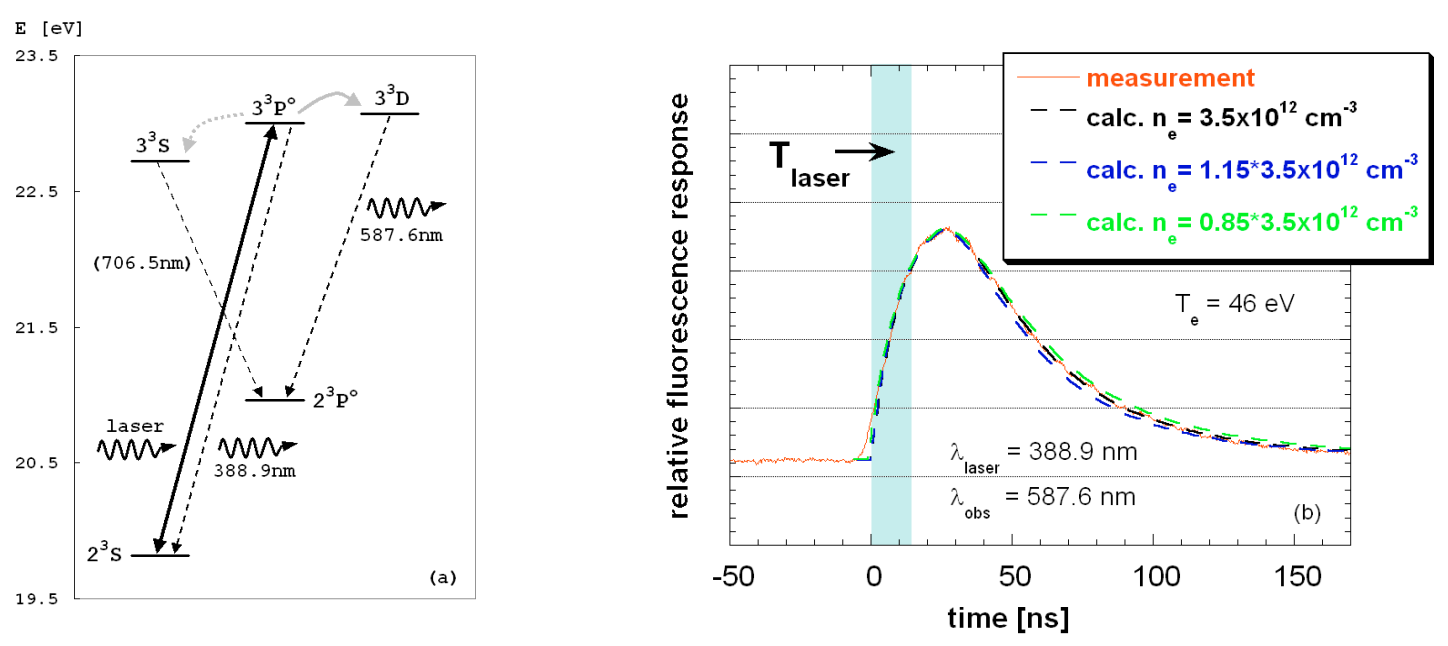

FIGURE 1. (a) Scheme of the laser excitation and fluorescence observation for the derivation of the electron density. (b) Best fit of the measured collision-induced fluorescence signal time trace originating from the level $3^{3} \mathrm{D}(\lambda=587.6 \mathrm{~nm})$ upon the laser excitation at $\lambda=388.9 \mathrm{~nm}$. Two additional fits are overlaid obtained by enhancing and lowering the electron density by $15 \%$. The measurement is performed in situ in the tokamak. 
The sensitivity of the decay shape of the considered collision-induced signal to the change of electron density will of course depend on the density itself and also on the temperature. In Figure 2 and Figure 3 simulations of the time traces of the fluorescence signals at the laser wavelength $\lambda=388.9 \mathrm{~nm}$ as well as at $\lambda=587.6 \mathrm{~nm}$ for five different pairs of $n_{\mathrm{e}}$ and $T_{\mathrm{e}}$ are shown. In Figure 2 (a) the signals are rescaled to the maximum value and shifted in time such that the maximum signals appear at the same point in time. It is done in this way in order to be able to compare the signal shapes starting approximately from the maximum (which is after the end of the laser pulse) since they are then independent from the temporal profile of the laser power. Considerable shortening of the decay times is visible with increasing parameter values (this is because the excessive upper population finds with increasing collisional transfer to the other $n=3$ - 4 triplet levels additional decay channels to the levels of the $n=2$ shell). The method becomes more sensitive when fitting the whole time traces including the rising edges. This can be done if the temporal profile of the laser saturation parameter (i.e. also the spatial distribution of the spectral laser power density at the observation volume) is known since it will influence the rising part of the fluorescence profile. However, this requires additional calibration and adjustment effort.
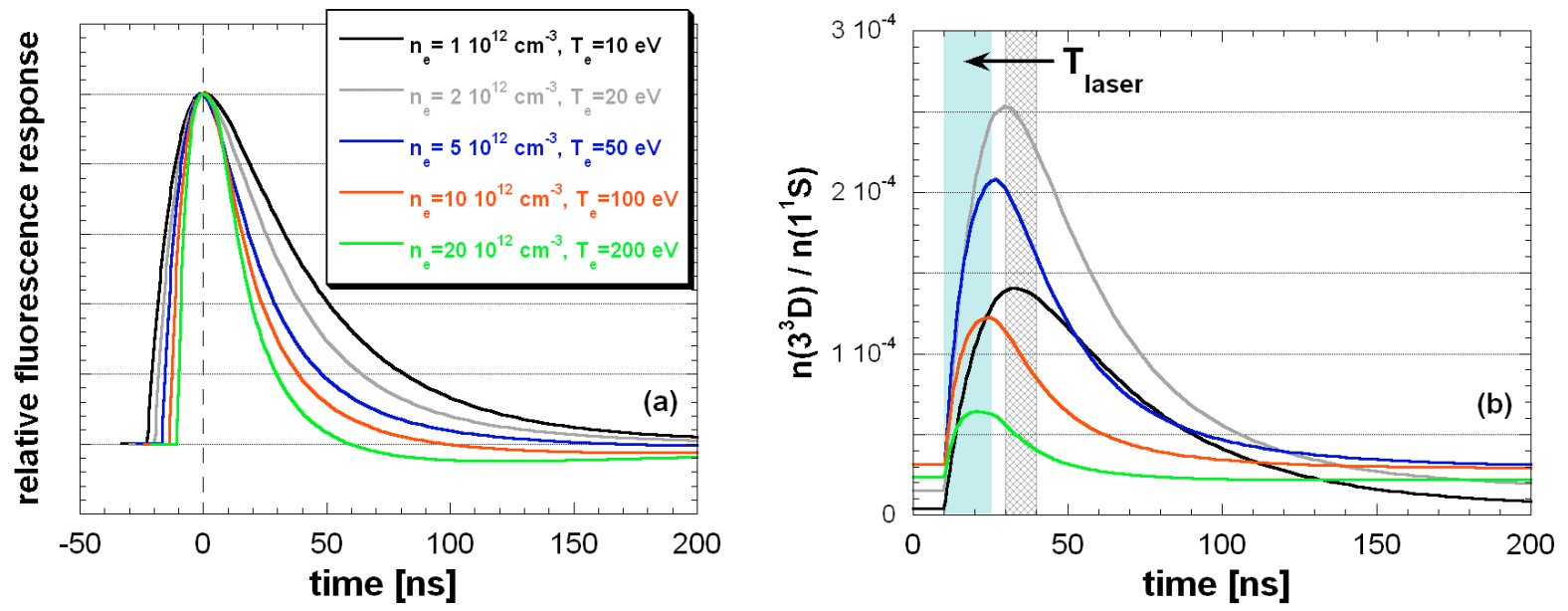

FIGURE 2. Simulation of the relative fluorescence response at $\lambda=587.6 \mathrm{~nm}$ as a result of a laser pulse at $\lambda=388.9 \mathrm{~nm}$ performed for five different pairs of $\left(n_{\mathrm{e}}, T_{\mathrm{e}}\right)$. (a) Resulting curves rescaled in the amplitude and shifted in the time according to the maximum. (b) Resulting curves without rescaling and shift in time.

The maximum signal amplitudes of both signals at the laser wavelength and the one induced by collisions are strongly correlated to the initial population density of the lower level $2^{3} \mathrm{~S}$, which has a maximum at electron temperatures of $T_{\mathrm{e}} \cong 20-50 \mathrm{eV}$ depending on the value of $n_{\mathrm{e}}$. This is demonstrated for the collision-induced signal in FIGURE 2 (b) and for the signal at the laser wavelength in FIGURE 3 (a) - the values of population densities relative to the ground state population are plotted without rescaling. The population increase is getting lower for $\mathrm{T}_{\mathrm{e}}>20 \mathrm{eV}$. Hence, at higher temperatures the signals will become poorer and the fits less reliable. In this case additional analysis by using the ratio of the time integrated laser- and collision-induced fluorescence signals can be applied [2]. (From the experimental point of view this additional measurement would require a spatial separation of fluorescence photons at both wavelengths e.g. by a beam splitter and simultaneous recording of both signals by two relatively calibrated detectors; absolute signal measurement connected with severe experimental effort and additional uncertainties is not necessary). In FIGURE 3 (b) the time traces of the ratios of the collisional- and the laser-induced fluorescence signals for the same pairs of $n_{\mathrm{e}}$ and $T_{\mathrm{e}}$ are plotted. The gray box indicates a possible integration time interval of the signal after the end of the laser pulse (including the signals during the laser pulse would increase the signal-to-noise ratio provided the laser stray light can be sufficiently suppressed). As we see the signal ratio reveals a pronounced dependence on the chosen plasma parameters. In the case of a too high level of scattered laser light, the fluorescence from the level $3^{3} \mathrm{~S}$ at $\lambda=706.5 \mathrm{~nm}$ (see FIGURE 1 (a)) instead of that at the laser wavelength could be used [7].

However, there are some limitations to the derivation of the electron density as described above. The collisional transfer rate between the level $3^{3} \mathrm{P}^{\mathrm{o}}$ and $3^{3} \mathrm{D}$ depends explicitly on the electron density and the rate coefficient, which is a weak function of electron temperature. It drops by a factor of two with a temperature change from 20 to $200 \mathrm{eV}$. Though a corresponding increase of the electron density by a factor of ten has a much stronger impact on the 
transfer rate - it increases by the same factor of ten, the consequence is that the electron density cannot be determined from the fluorescence signal without a certain knowledge of electron temperature. Additional simulations have shown that for a desired accuracy of the $n_{\mathrm{e}}$ measurement of $10 \%, T_{\mathrm{e}}$ must be known with an accuracy of $30-40 \%$, depending on $n_{\mathrm{e}}$ and $T_{\mathrm{e}}$. For this reason this method makes sense only if accompanied by another diagnostic. The use of atomic helium line radiation observed passively naturally seems to be the first choice because of the intrinsic necessity for the existence of neutral helium in the observation volume of the LIF diagnostics.
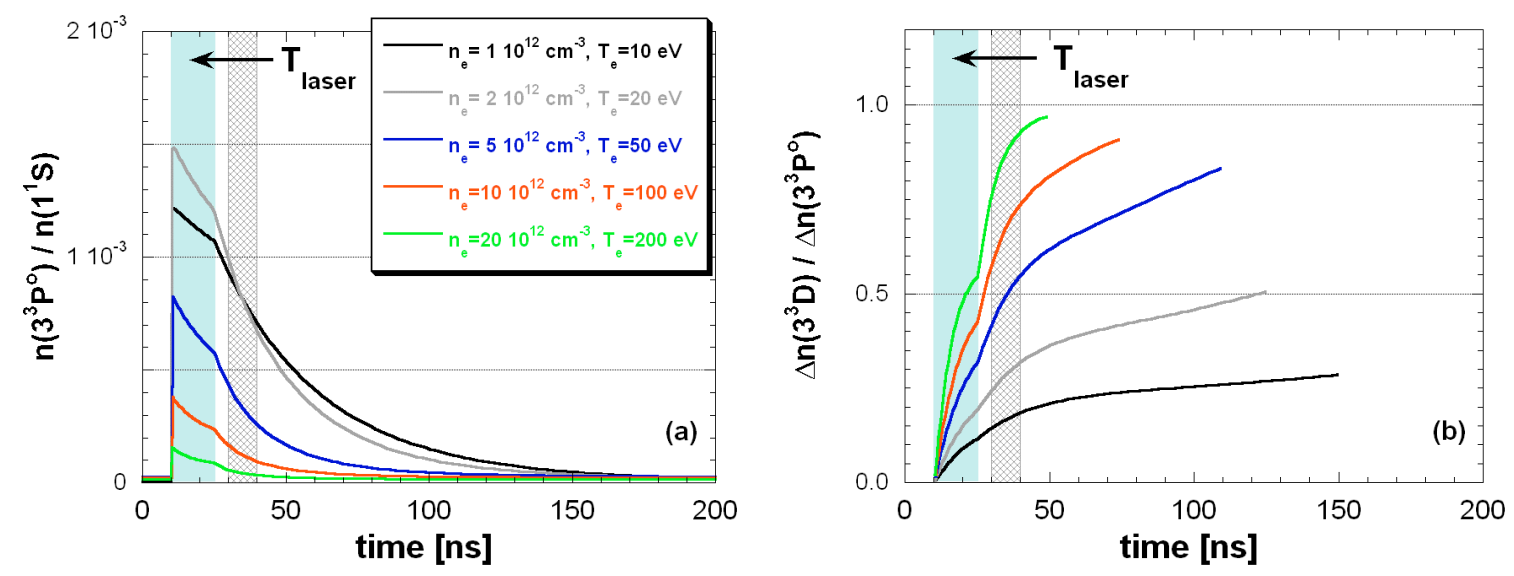

FIGURE 3. (a) Calculation of the relative fluorescence response at the laser wavelength as a result of a laser pulse at $\lambda=388.9 \mathrm{~nm}$ performed for five different pairs of $\left(n_{\mathrm{e}}, T_{\mathrm{e}}\right)$. (b) Calculated ratio of the collisional- and laser-induced fluorescence signals upon the same laser pulse as in (a).

Another limitation is the noise level of the fluorescence signals. When using a thermal or supersonic helium beam as neutral helium source the helium density will decrease along the beam path towards the plasma center due to ionization processes up to a complete vanishing of the beam. Hence, a similar intrinsic radial limitation of the measurement method appears as in the case of the $n_{\mathrm{e}}$ and $T_{\mathrm{e}}$ derivation from line intensity ratios on helium beams. A possible signal enhancement in comparison to our experimental setup could be achieved e.g. by the use of a laser with a larger spectral width covering the whole Zeeman structure $\left(B_{T} \approx 2.5 \mathrm{~T}\right)$ or, better, by light collection over the full toroidal extension of the diverging helium beam [6]. On the other hand, the number of laser pulses for signal averaging can be increased. The measured time trace shown in FIGURE 1 (b) was averaged over 20 pulses. At a laser repetition frequency of $150 \mathrm{~Hz}$ such a measurement takes 0.13 seconds. However, a single sample takes only few hundred nanoseconds. This is seen in FIGURE 2 (b) and FIGURE 3 (a). After two hundred nanoseconds the laser-perturbed level populations are nearly relaxed i.e. their initial values are again established and the system is in principle ready for the next sample. The use of a dye laser provides very large saturation parameters of $S \gg 1000$ in the visible spectral range so that one laser pulse could be divided in several subpulses with still enough power density. The subpulses would additionally have to be delayed each one by a time interval of $t_{\text {delay }}>200 \mathrm{~ns}$. Averaging the fluorescence signals upon the subpulses would preserve the signal-to-noise ratio of the case when using several undivided pulses and reduce the measurement time to a few microseconds.

\section{REFERENCES}

1. P. Chall, E.K. Souw and J. Uhlenbusch, J. Quant. Spectrosc. Radiat. Transfer 34, 309-320 (1985).

2. K. Tsuchida, S. Miyake, K. Kadota and J. Fujita, Plasma Physics 25, 991-999 (1983).

3. D.A. Shcheglov, S.I. Vetrov, I.V. Moskalenko, A.A. Skovoroda and D.A. Shuvaev, Plasma Physics Reports 32, 119-122 (2006).

4. E. Hintz and B. Schweer, Plasma Phys. Control. Fusion 37, A87-A101 (1995).

5. M. Brix, "Messung von Elektronentemperatur und -dichte mittels Heliumstrahldiagnostik im Randschichtplasma eines Tokamaks", Jülicher Report Jül-3638 (1998).

6. M. Krychowiak et al., "LIF measurements on an atomic helium beam in the edge of a fusion plasma - spectroscopic analysis and first results", submitted to Plasma Phys. Control. Fusion.

7. I.V. Moskalenko et al., Plasma Devices and Operations 12, 247-258 (2004). 